STUDI

FRANCESI

\section{Studi Francesi}

Rivista quadrimestrale fondata da Franco Simone

185 (LXII | II) | 2018

OCTAVE MIRBEAU: UNE CONSCIENCE AU TOURNANT

DU SIEECLE - sous la direction de Ida Merello

\title{
GILLES BONNET, Pour une poétique numérique. Littérature et internet
}

Roberta Sapino

\section{(2) OpenEdition \\ Journals}

\section{Edizione digitale}

URL: http://journals.openedition.org/studifrancesi/14349

DOI: $10.4000 /$ studifrancesi. 14349

ISSN: 2421-5856

\section{Editore}

Rosenberg \& Sellier

\section{Edizione cartacea}

Data di pubblicazione: 1 août 2018

Paginazione: 384-385

ISSN: 0039-2944

\section{Notizia bibliografica digitale}

Roberta Sapino, «GILLES BONNET, Pour une poétique numérique. Littérature et internet», Studi Francesi [Online], 185 (LXII | II) | 2018, online dal 01 septembre 2019, consultato il 13 janvier 2021. URL: http:// journals.openedition.org/studifrancesi/14349; DOI: https://doi.org/10.4000/studifrancesi. 14349

Questo documento è stato generato automaticamente il 13 janvier 2021.

\section{(c) (i) (9)}

Studi Francesi è distribuita con Licenza Creative Commons Attribuzione - Non commerciale - Non opere derivate 4.0 Internazionale. 


\title{
GILLES BONNET, Pour une poétique numérique. Littérature et internet
}

\author{
Roberta Sapino
}

\section{NOTIZIA}

GILLES BONNET, Pour une poétique numérique. Littérature et internet, Hermann, 2017, 368 pp.

1 Tra i tanti libri pubblicati recentemente sulle cosiddette digital humanities, il saggio di Gilles BONNET spicca per la prospettiva essenzialmente letteraria, se non poetica, attraverso la quale l'autore osserva il mondo digitale. Lo scopo della ricerca è di esplorare le zone di incontro tra la "cultura del libro" e la "cultura dello schermo", tra la teoria letteraria ormai consolidata e i principi fondamentali della cultura digitale, al fine di porre le basi (senza tuttavia ambire a elaborarne a fondo i termini) di una $e$ poétique, una poetica capace da un lato di considerare il testo digitale in rapporto alla storia e alla teoria della letteratura, dall'altro di valorizzarne gli elementi di peculiarità. Come già avvenuto in passato, in concomitanza con la diffusione di grandi innovazioni tecnologiche e sociali, lo scrittore del ventunesimo secolo si trova a rinegoziare il suo statuto e la sua identità. Soltanto qualora egli non si limiti a sfruttare le risorse del web per promuovere l'opera cartacea, ma assuma invece un atteggiamento di "engagement constitutif" nei confronti dello spazio digitale, confrontandosi attivamente con i limiti e le potenzialità del mezzo tecnologico e impegnandosi a farne uno strumento nuovo di creazione letteraria, egli può dirsi (secondo una definizione che Bonnet rielabora a partire da Barthes) «pleinement écrivain». È a questo scrittore ideale, capace di manipolare in maniera originale le imposizioni dell'ambiente digitale per difendere la letterarietà dalle minacce della massificazione, che Bonnet attribuisce il nome di «écranvain» (Introduction, pp.7-18). Diviso in tre sezioni, il volume mette in luce la vitalità di uno spazio letterario in dialogo costante tanto con la storia letteraria precedente che con le innovazioni tecnologiche (e, conseguentemente, formali) proiettate verso il futuro. 
2 La prima sezione è intitolata «Seuil» e si concentra sulla messa in discussione dei limiti imposti all'opera letteraria dalle convenzioni legate al mezzo e al genere. Soffermandosi su alcuni casi in cui l'immagine assume il ruolo di Image-seuil (pp. 21-73), come i «nanodrames» di Jean-François Magre o il blog «L'œil ouvert» di Ossiane Océane, Bonnet considera la scelta di comporre testi a partire da un'immagine come uno sviluppo contemporaneo della scrittura à contrainte. La fotografia, privata dell'aura in virtù della sua infinita riproducibilità e modificabilità, perde il suo valore di documento del reale per diventare testimonianza di uno sguardo soggettivo e di un processo di creazione che perdura nel tempo. D'altro canto, proprio l'abbondanza di immagini che determina lo stato di «obscenité du réel» contemporaneo, in cui tutto è potenzialmente esposto e visibile, induce l'écranvain a cercare forme fondate sull'opacità e l'astrazione, per rielaborare in maniera nuova l'antico legame tra la fotografia e la spettralità. La possibilità di rielaborare potenzialmente all'infinito la materia iconologica e letteraria fa dell'opera digitale quello che Bonnet definisce un «hypéritexte» (L'œuvre mobile: vers l'hypéritexte, pp. 81-110), fondato su una pratica non solo di conservazione, ma di rilettura e riappropriazione. La trasposizione di Limite dall'edizione cartacea (Minuit, 1985) al sito «Tiers Livre» operata da François Bon offre un esempio eclatante di riappropriazione dell'opera sia attraverso l'aggiunta di elementi paratestuali assenti dall'edizione cartacea, sia con l'interpolazione di passi interamente nuovi all'interno del testo stesso.

3 La seconda parte, «Soi», considera le diverse forme di scrittura di sé offerte dallo spazio digitale. Se, come scriveva Lejeune, «le sujet profond de l'autobiographie, c'est le nom propre», allora siti con nomi come www.maulpoix.net o www.lesliekaplan.net rientreranno, secondo Bonnet, nel campo dell'autobiografia (L'autoblographie, pp.113-171). In particolare, la struttura del blog riattiva alcune pratiche della pragmatica epistolare come la datazione dei messaggi e l'enunciazione personale, mentre la "tendenza antologica" tipica del digitale (sollecitata dall'enorme capacità dei supporti informatici di contenere dati) si declina - come per esempio nel sito di Nicole Caligaris - in liste e assemblaggi di citazioni, modelli, influenze, fino a costituire una vera e propria «allo-auto-biographie». D'altro canto, la struttura del blog o del sito internet, basata meno sulla linearità e più sulla dispersione di tracce unite da link più o meno solidi, e la possibilità di far coincidere sulla stessa pagina sistemi semiotici diversi, nonché testi realizzati in momenti temporali anche molto distanti tra loro, conferisce una struttura visiva all'abbandono dell'illusione di poter offrire un'immagine globale e unitaria di sé, rimettendo in discussione i principi fondamentali dell'autobiografia. Così rielaborato, conclude Bonnet, il regime autobiografico digitale entra nel regime dell'illusorio, inscrivendosi nella scia di opere come Enfance di Nathalie Sarraute. Un caso particolare di scrittura di sé, comparabile in parte al racconto di viaggio o al diario di bordo, è Le blog de résidence (il diario digitale che l'autore sceglie di tenere in occasione di soggiorni di scrittura in luoghi diversi da quelli abituali) che viene analizzato nel dettaglio nella seconda parte di questa sezione (pp. 173-216).

4 «Savoir» è il titolo della terza sezione, in cui il mezzo digitale è osservato in relazione a due elementi molto diversi tra loro, ma ugualmente fondamentali nella costruzione dello spazio letterario: da un lato il genere letterario del saggio, dall'altro la "casa dello scrittore" concepita come una sorta di museo della persona e dell'opera. Per la maggior parte degli scrittori presenti sul web, osserva Bonnet, Internet si presenta non come il 
luogo di pubblicazione primario, ma come un suo accompagnamento. In questo spazio, l'autore può assumere a pieno il ruolo di écrivain-lecteur, sia corredando le proprie opere di commenti, paratesti, annotazioni varie, immagini di manoscritti e qualsiasi elemento possa contribuire alla discussione attiva dei testi, sia rendendo conto delle proprie letture di testi altrui, originando così una sorta di museo immaginario delle sue fonti di ispirazione. Quest'insieme di testi costantemente rielaborati e di idee riformulate senza sosta compone quello che Bonnet definisce l'e-ssai (pp. 219-263). In maniera analoga, in quanto si configura come una sorta di "archivio di sé", il sito realizzato dall'écranvain può essere considerato come l'espressione di un intento museale nato dalla rielaborazione dell'immagine di Internet come «archivio dell'umanità» (La maison d'écranvain, pp. 265-328). Il sito Internet diventa allora un luogo di memoria con valore patrimoniale, sia quando è realizzato da uno scrittore vivente, sia quando (come nel caso delle piattaforme dedicate a André Breton o a SaintJohn Perse) è dedicato a un autore scomparso.

5 Se la letteratura ha tra le sue funzioni quella di testimoniare i cambiamenti epistemologici in atto, accordandovisi o criticandoli radicalmente, ma senza mai mantenersi immune, allora essa non può esimersi dal confronto con l'«ecosistema digitale» che coinvolge ormai tutti gli aspetti dell'esistenza umana (Conclusion. L'écosystème numérique, pp.329-334). L'elaborazione di una "poetica digitale" trova allora piena legittimità, e potrebbe aprire modalità interpretative interessanti per lo studio della letteratura contemporanea in senso lato. 